Article

\title{
Refined Expected Value Decision Rules under Orthopair Fuzzy Environment
}

\author{
Yige Xue and Yong Deng * \\ Institute of Fundamental and Frontier Sciences, University of Electronic Science and Technology of China, \\ Chengdu 610054, China; 2016200301019@std.uestc.edu.cn \\ * Correspondence: dengentropy@uestc.edu.cn or prof.deng@hotmail.com
}

Received: 17 February 2020; Accepted: 13 March 2020; Published: 18 March 2020

\begin{abstract}
Refined expected value decision rules can refine the calculation of the expected value and make decisions by estimating the expected values of different alternatives, which use many theories, such as Choquet integral, PM function, measure and so on. However, the refined expected value decision rules have not been applied to the orthopair fuzzy environment yet. To address this issue, in this paper we propose the refined expected value decision rules under the orthopair fuzzy environment, which can apply the refined expected value decision rules on the issues of decision making that is described in the orthopair fuzzy environment. Numerical examples were applied to verify the availability and flexibility of the new refined expected value decision rules model. The experimental results demonstrate that the proposed model can apply refined expected value decision rules in the orthopair fuzzy environment and solve the decision making issues with the orthopair fuzzy environment successfully.
\end{abstract}

Keywords: refined expected value; orthopair fuzzy environment; decision rules

\section{Introduction}

In the real world, there are many uncertainties and unreliabilities [1-4]. In order to deal with the uncertainties [5-7], many mathematical theories are proposed, such as Bayesian network [8], hyper structures [9], fuzzy sets (FS) [10-12], hesitant fuzzy subalgebras [13], D-S evidence theory [14-16], information quality [17,18], Z-number [19,20], D-number [21], entropy [22,23] and belief structure [24] and are applied in many fields [25-27]. Among these theories and models, the orthopair fuzzy set (OFS) [28-30] allows the membership degree, non-membership degree and hesitancy degree to be $[0,1] \times[0,1]$, which results in the orthopair fuzzy set generalization of the intuitionistic fuzzy set and Pythagorean fuzzy set and giving great freedom to the modelers of systems in order to capture human knowledge. In this way, the orthopair fuzzy set is able to deal with the uncertainties more flexibly and accurately, has been widely applied in many fields [31,32], such as uncertainty multi-attribute decision making [33], enterprise resource planning systems selection [34], potential evaluation of emerging technology commercialization [35], green suppliers selection [36], scheme selection of construction project [37], venture capital in real estate market [38], medical diagnosis [39] and so on.

Recently, Yager proposed the refined expected value decision rules, which has the promising aspect [40]. However, what the refined expected value decision rules for a given orthopair fuzzy set is still an open issue to be addressed.

This paper proposes refined expected value decision rules under orthopair fuzzy environment, which is an approach that can refine the expected values of alternatives under orthopair fuzzy environment and make decision. It means that if an issue of decision making is under an orthopair fuzzy environment, then the refined expected value decision rules can be applied to solve this kind of issue. 
The remain of this paper is structured as follows. Section 2 introduces the preliminary details. Section 3 presents the refined expected value decision rules under the orthopair fuzzy environment. Section 4 illustrates the flexibility and accuracy of the refined expected value decision rules under an orthopair fuzzy environment. Section 5 summarizes the whole paper.

\section{Preliminaries}

To deal with uncertainty, many tools and models have been proposed [41-44], such as fuzzy sets [45,46], basic probability assignment [47,48], rough sets [49], ordered weighted average operator [50], entropy [51-54], game theory [55,56] and complex networks [57-62]. In this section, some relative definitions are briefly introduced, such as refined expected value [40], measure [63,64] and Choquet integral [65].

\subsection{Aggregation Function}

The definition of aggregation function is defined as follows:

Definition 1. (Aggregation Function) [66]

A mapping Agg: $[0,1]^{n} \rightarrow[0,1]$ is an aggregation function if

$$
\begin{gathered}
\operatorname{Agg}(0, \ldots, 0)=0 \\
\operatorname{Agg}\left(c_{1}, \ldots, c_{n}\right) \geq \operatorname{Agg}\left(d_{1}, \ldots, d_{n}\right) \text { if } 1 \geq c_{j} \geq d_{j} \geq 0 \text { for all } j \\
\operatorname{Agg}(1, \ldots, 1)=1
\end{gathered}
$$

Aggregation functions have many examples and models. For example, $\operatorname{Agg}\left(c_{1}, \ldots, c_{n}\right)=\prod_{n}^{i=1} c_{i}$ and $\operatorname{Agg}\left(c_{1}, \ldots, c_{n}\right)=\operatorname{Min}_{i}\left[c_{1}, \ldots, c_{n}\right]$ are aggregation functions.

\subsection{Measure}

The real world is uncertain. So, how to measure the degree of uncertainty is a important issue. The measure has effective performance in this issue. Given a space $Y$, the definition of measure is defined as follows:

Definition 2. (Measure) [67]

Assume $\mu$ on $Y$ is a mapping $\mu: Y \rightarrow[0,1]$. If $\mu$ satisfies the following conditions:

$$
\begin{gathered}
\mu(\varnothing)=0 \\
\mu(C) \geq \mu(D) \text { if } D \subseteq C \\
\mu(Y)=1
\end{gathered}
$$

where, $C$ and $D$ are subsets of $Y$.

Hence, $\mu$ is a measure.

As a highly effective tool to indicate uncertainty, measure has wide applications [68], which is very flexible and effective.

\subsection{Dual}

Given a measure $\mu$ on a space $Y$, the definition of its dual $\hat{\mu}$ is defined as follow:

Definition 3. (Dual) [69]

$$
\hat{\mu}(F)=1-\mu(\bar{F})
$$


$\hat{\mu}$ is also a measure. The self-dual is a special measure such that $\hat{\mu}(F)=\mu(F)$.

\subsection{Choquet Integral}

Given a measure $\mu$ on a space $Y=\left\{y_{1}, \ldots, y_{n}\right\}$. The definition of Choquet integral Choq $\mu$ related to $\mu$ is defined as follows:

Definition 4. (Choquet Integral) [70]

$$
\operatorname{Choq}_{\mu}\left(y_{1}, \ldots, y_{n}\right)=\sum_{j=1}^{n}\left(\mu\left(H_{j}\right)-\mu\left(H_{j-1}\right)\right) y_{\alpha(j)}
$$

where $\alpha(j)$ is an index function and $y_{\alpha(j)}$ is the jth largest element in $2^{Y}$ such that $y_{\alpha(n)} \leq \cdots \leq y_{\alpha(2)} \leq y_{\alpha(1)}$ and $H_{j}=\left\{y_{\alpha(i)}\right.$ for $i=1$ to $\left.j\right\}$ is subset of $2^{Y}$.

\subsection{Primal Monotonic Function}

Definition 5. (Primal Monotonic Function) [71]

A function $f:[0,1] \rightarrow[0,1]$ is called a primal monotonic function, PM function, if

$$
\begin{gathered}
f(0)=0 \\
f(A) \leq f(B) \text { if } 0 \leq A \leq B \leq 1 \\
f(1)=1
\end{gathered}
$$

For example, $f(x)=x^{r}$ for $r \in(0, \infty)$ is a PM function.

\subsection{Refined Expected Value}

Assume a collection of alternatives is $B=\left\{B_{1}, B_{2}, \cdots, B_{n}\right\}$, each $B_{i}$ with probability distribution $\operatorname{Prob}_{i}$ on a space $Z=\left\{z_{1}, z_{2}, \cdots, z_{m}\right\}$ is an uncertain alternative and $f$ is a PM function.

The definition of refined expected value related to alternative $B_{i}$ is defined as follows:

Definition 6. (Refined Expected Value) [40]

$$
E V_{f}\left(\operatorname{Prob}_{i}\right)=\sum_{j=1}^{m}\left(f\left(\operatorname{Prob}_{i}\left(H_{j}\right)\right)-f\left(\operatorname{Prob}_{i}\left(H_{j-1}\right)\right)\right) z_{j}
$$

Here $z_{j}$ is the jth largest element in Z. Hence, $\operatorname{Prob}_{i}\left(z_{1}\right) \geq \operatorname{Prob}_{i}\left(z_{2}\right) \geq \cdots \geq \operatorname{Prob}_{i}\left(z_{n}\right)$ and $H_{j}=\left\{z_{k}\right.$ for $k=1$ to $\left.j\right\}$.

\subsection{Generalized Orthopair Fuzzy Sets}

Dealing with uncertainty is an open issue and many tools are presented to address this issue [72,73]. Generalized orthopair fuzzy sets have extended intuitionistic fuzzy sets [74] and Pythagorean fuzzy sets [75,76]. The orthopair fuzzy sets have advantages in representing uncertainties [77] and have been used in a wide scope of applications [78,79]. It is more flexible, practical and efficient than intuitionistic fuzzy sets and Pythagorean fuzzy sets in dealing with ambiguity and uncertainty $[80,81]$.

Given an universe set $Z=\left\{z_{1}, z_{2}, \cdots, z_{n}\right\}$, the definition of q-rung orthopair fuzzy set $E$ on $Z$ is defined as follows: 
Definition 7. (Q-Rung Orthopair Fuzzy Set) [28]

$$
E=\left\{\left\langle z_{j}, E^{+}\left(z_{j}\right), E^{-}\left(z_{j}\right)\right\rangle_{q}: z_{j} \in Z\right\}
$$

where $E\left(z_{j}\right)=\left\langle E^{+}\left(z_{j}\right), E^{-}\left(z_{j}\right)\right\rangle_{q}$ is a q-rung orthopair membership grade [28]. If $q=2$, E will be a Pythagorean fuzzy set. If $q=1, E$ will be an intuitionistic fuzzy set.

Since each orthopair fuzzy grade consists of three degrees, which are membership degree, non-membership degree and hesitancy degree. It is not easy for an orthopair fuzzy grade to compare with each other. Therefore, if we can change the three degrees of an orthopair fuzzy value into a degree, we can make decisions easily. So, assume $E(z)=\left\langle E^{+}(z), E^{-}(z)\right\rangle_{q}$ is a given q-rung orthopair fuzzy grade, Yager has proposed the following [82]:

$$
V(z)=\left(\lambda\left(B_{U}(z)\right)^{q}+(1-\lambda)\left(B_{L}(z)\right)^{q}\right)^{1 / q}
$$

where, $B_{L}(z)=E^{+}(z)$ and $B_{U}(z)=\left(1-\left(E^{-}(z)\right)^{q}\right)^{1 / q}$.

\section{The Proposed Model}

In this paper, the refined expected value decision rules under orthopair fuzzy environment is proposed. The refined expected value decision rules is a good tool to represent uncertainty, but it has been applied under orthopair fuzzy environment, which is still an open issue. In this section, the refined expected value decision rules under orthopair fuzzy environment has been proposed, which can solve the problem of decision making under the orthopair fuzzy environment. The refined expected value decision rules under orthopair fuzzy environment can get an interval value from an object or alternative with the aid of Choquet integral, primal monotonic function and refined expected value. It leads to the result that interval values can be obtained by membership degree, non-membership degree and hesitancy degree under orthopair fuzzy environment to indicate the uncertain information of an object. It means that the proposed model can use fully the information of orthopair fuzzy environment to make decision.

Definition 8. (Refined expected value decision rules under orthopair fuzzy environment)

Given an orthopair fuzzy set $E=\left\{\left\langle z_{j}, E^{+}\left(z_{j}\right), E^{-}\left(z_{j}\right)\right\rangle_{q}: z_{j} \in Z\right\}$ with orthopair fuzzy grades $E\left(z_{j}\right)=\left\langle E^{+}\left(z_{j}\right), E^{-}\left(z_{j}\right)\right\rangle_{q}$ such that $\left(E^{+}\left(z_{j}\right)\right)^{q}+\left(E^{-}\left(z_{j}\right)\right)^{q} \leq 1$. Assume $f$ is a PM function and $\mu$ is a measure on $\mathrm{Z}$.

The refined expected value decision rules under orthopair fuzzy environment is defined as follows:

$$
\begin{aligned}
& E^{+}(z)=\operatorname{Choq}_{\mu}\left(E^{+}\left(z_{1}\right), \ldots, E^{+}\left(z_{n}\right)\right) \\
&= \sum_{j=1}^{n}\left(f\left(\mu\left(G_{j}\right)\right)-f\left(\mu\left(G_{j-1}\right)\right)\right) E^{+}\left(z_{\alpha(j)}\right) \\
& E^{-}(z)=\operatorname{Neg}_{q}\left(\operatorname{Choq}_{\mu}\left(\operatorname{Neg}_{q}\left(E^{-}\left(z_{1}\right)\right), \ldots, N_{e g}\left(E^{-}\left(z_{n}\right)\right)\right)\right)
\end{aligned}
$$

Then, $\left\langle E^{+}(z), E^{+}(z)\right\rangle$ is an orthopair value.

Where, $G_{j}=\left\{z_{\alpha(1)}, z_{\alpha(2)}, \ldots, z_{\alpha(j)}\right\}$ and $\operatorname{Neg}_{q}(d)=\left(1-d^{q}\right)^{1 / q}$.

Where $\mu$ is a cardinality-based measure and $f$ is linear function, $f(x)=x$. Here, we have a set of parameters $0=a_{0} \leq a_{1} \ldots \leq a_{n}=1$ such that $\mu\left(G_{j}\right)=a_{\left|G_{j}\right|}$. The value of $\mu\left(G_{j}\right)$ is decided on the cardinality of the set $G_{j}$. We denote $v_{j}=a_{j}-a_{j-1}$ so that $w_{j}=f\left(\mu\left(G_{j}\right)\right)-f\left(\mu\left(G_{j-1}\right)\right)=$ $\mu\left(G_{j}\right)-\mu\left(G_{j-1}\right)=a_{j}-a_{j-1}=v_{j}$. We see this is essentially an OWA aggregation [83]. Based on the 
above assumptions, we provide some important and interesting theorems about the refined expected value under orthopair fuzzy environment.

Theorem 1. Given a q-rung orthopair fuzzy set $E=\left\{\left\langle z_{j}, E^{+}\left(z_{j}\right), E^{-}\left(z_{j}\right)\right\rangle_{q}: z_{j} \in Z\right\}$ with orthopair fuzzy grades $E\left(z_{j}\right)=\left\langle E^{+}\left(z_{j}\right), E^{-}\left(z_{j}\right)\right\rangle_{q}$ and $E^{+}\left(z_{j}\right)$ and $E^{-}\left(z_{j}\right)$ as defined above, then $\left(E^{+}(z)\right)^{q}+$ $\left(E^{-}(z)\right)^{q} \leq 1$.

Proof. Since the Choquet integral related to a cardinality-based measure is OWA aggregation [83].

Hence, we can get that $E^{+}(z)$ and $E^{-}(z)$ are OWA aggregation.

Since each $E(z)$ has $\left(E^{+}(z)\right)^{q}+\left(E^{-}(z)\right)^{q} \leq 1$

Then

$$
\begin{gathered}
E^{+}(z)=\operatorname{Agg}\left(E^{+}\left(z_{1}\right), \ldots, E^{+}\left(z_{n}\right)\right) \\
E^{-}(z)=\left(1-\operatorname{Agg}\left(\left(1-\left(E^{-}\left(z_{1}\right)\right)^{q}\right)^{1 / q}, \ldots,\left(1-\left(E^{-}\left(z_{n}\right)\right)^{q}\right)^{1 / q}\right)^{q}\right)^{1 / q}
\end{gathered}
$$

Since $\left(E^{+}\left(z_{i}\right)\right)^{q} \leq 1-\left(E^{-}\left(z_{i}\right)\right)^{q}$, so

$$
E^{+}(z) \leq \operatorname{Agg}\left(\left(1-\left(E^{-}\left(z_{1}\right)\right)^{q}\right)^{1 / q}, \ldots,\left(1-\left(E^{-}\left(z_{n}\right)\right)^{q}\right)^{1 / q}\right)
$$

Here, we see that $\left(E^{+}(z)\right)^{q}+\left(E^{-}(z)\right)^{q} \leq 1$.

Hence, we know that $\left\langle E^{+}(z), E^{-}(z)\right\rangle$ is an q-rung orthopair fuzzy grade.

When $\mu$ is a probability measure and $f$ is linear function, $f(x)=x$, we provide some important and interesting theorems about the refined expected value under orthopair fuzzy environment.

Theorem 2. If $\left\langle E^{+}(z), E^{-}(z)\right\rangle$ is a refined expected value under orthopair fuzzy environment, then

$$
\left\langle E^{+}(z), E^{-}(z)\right\rangle=\left\langle\sum_{i=1}^{n} p_{i} E^{+}\left(z_{i}\right),\left(1-\left(\sum_{j=1}^{n} p_{i}\left(1-E^{-}\left(z_{i}\right)^{q}\right)^{1 / q}\right)^{q}\right)^{1 / q}\right\rangle
$$

Proof. Relying on Equation (15), Equation (16) and arising from the definition of the refined expected value decision rules under orthopair fuzzy environment, one has the following equation:

$$
\begin{aligned}
E^{+}(z) & =\operatorname{Choq}_{\mu}\left(E^{+}\left(z_{1}\right), \ldots, E^{+}\left(z_{n}\right)\right) \\
& =\sum_{i=1}^{n}\left(f\left(\mu\left(G_{i}\right)\right)-f\left(\mu\left(G_{i-1}\right)\right)\right) E^{+}\left(z_{\alpha(i)}\right) \\
& =\sum_{i=1}^{n} p_{\alpha(i)} E^{+}\left(z_{\alpha(i)}\right) \\
& =\sum_{i=1}^{n} p_{i} E^{+}\left(z_{i}\right)
\end{aligned}
$$




$$
\begin{aligned}
E^{-}(z) & =\operatorname{Neg}_{q}\left(\operatorname{Choq}_{\mu}\left(\operatorname{Neg}_{q}\left(E^{-}\left(z_{1}\right)\right), \ldots, \operatorname{Neg}_{q}\left(E^{-}\left(z_{n}\right)\right)\right)\right) \\
& =\left(1-\operatorname{Choq}_{\mu}\left(\operatorname{Neg}_{q}\left(E^{-}\left(z_{1}\right)\right), \ldots, \operatorname{Neg}_{q}\left(E^{-}\left(z_{n}\right)\right)\right)^{q}\right)^{1 / q} \\
& =\left(1-\operatorname{Choq}_{\mu}\left(\left(1-E^{-}\left(z_{1}\right)^{q}\right)^{1 / q}, \ldots,\left(1-E^{-}\left(z_{n}\right)^{q}\right)^{1 / q}\right)^{q}\right)^{1 / q} \\
& =\left(1-\left(\sum_{i=1}^{n}\left(f\left(\mu\left(K_{i}\right)\right)-f\left(\mu\left(K_{i-1}\right)\right)\right)\left(1-E^{-}\left(z_{\vartheta(i)}\right)^{q}\right)^{1 / q}\right)^{q}\right)^{1 / q} \\
& =\left(1-\left(\sum_{i=1}^{n} p_{\vartheta(i)}\left(1-E^{-}\left(z_{\vartheta(i)}\right)^{q}\right)^{1 / q}\right)^{q}\right)^{1 / q} \\
& =\left(1-\left(\sum_{i=1}^{n} p_{i}\left(1-E^{-}\left(z_{i}\right)^{q}\right)^{1 / q}\right)^{q}\right)^{1 / q}
\end{aligned}
$$

So, we have that

$$
\left\langle E^{+}(z), E^{-}(z)\right\rangle=\left\langle\sum_{i=1}^{n} p_{i} E^{+}\left(z_{i}\right),\left(1-\left(\sum_{j=1}^{n} p_{i}\left(1-E^{-}\left(z_{i}\right)^{q}\right)^{1 / q}\right)^{q}\right)^{1 / q}\right\rangle
$$

Theorem 3. Assume $\left\langle E^{+}(z), E^{-}(z)\right\rangle$ is a refined expected value under orthopair fuzzy environment. Then, if Prob $=\left\{p_{j} \mid j=1, \ldots, n\right\}$ is a constant probability distribution such that $p_{i}=1$ for $j *$, then

$$
\left\langle E^{+}(z), E^{-}(z)\right\rangle=\left\langle E^{+}\left(z_{j *}\right), E^{-}\left(z_{j *}\right)\right\rangle
$$

Proof. In Theorem 2, we note that

$$
\left\langle E^{+}(z), E^{-}(z)\right\rangle=\left\langle\sum_{i=1}^{n} p_{i} E^{+}\left(z_{i}\right),\left(1-\left(\sum_{i=1}^{n} p_{i}\left(1-E^{-}\left(z_{i}\right)^{q}\right)^{1 / q}\right)^{q}\right)^{1 / q}\right\rangle
$$

For the definition of constant probability distribution, one has the equation as follows:

$$
p_{i}=1 \text { for } i=j *
$$

Based on Theorem 2, we get that

$$
\begin{aligned}
& E^{+}(z)=\sum_{i=1}^{n} p_{i} E^{+}\left(z_{i}\right)=E^{+}\left(z_{j *}\right) \\
E^{-}(z) & =\left(1-\left(\sum_{i=1}^{n} p_{i}\left(1-E^{-}\left(z_{i}\right)^{q}\right)^{1 / q}\right)^{q}\right)^{1 / q} \\
& =\left(1-\left(p_{j *}\left(1-E^{-}\left(z_{j *}\right)^{q}\right)^{1 / q}\right)^{q}\right)^{1 / q} \\
& =E^{-}\left(z_{j *}\right)
\end{aligned}
$$


Above all, we see that $\left\langle E^{+}(z), E^{-}(z)\right\rangle=\left\langle E^{+}\left(z_{j *}\right), E^{-}\left(z_{j *}\right)\right\rangle$. Obviously, the $\left\langle E^{+}(z), E^{-}(z)\right\rangle$ is also an orthopair fuzzy grade.

Example 1. Given an orthopair fuzzy set $Z=\left\{\left\langle z_{1}, 1,0\right\rangle_{q},\left\langle z_{2}, 0.4,0.3\right\rangle_{q},\left\langle z_{3}, 0.8,0.5\right\rangle_{q}\right\}$. Here, we see that

$$
\begin{gathered}
(1)^{q_{1}}+(0)^{q_{1}} \leq 1 \text { for } q_{1} \geq 1 \\
(0.4)^{q_{2}}+(0.3)^{q_{2}} \leq 1 \text { for } q_{2} \geq 1 \\
(0.8)^{q_{3}}+(0.5)^{q_{3}} \leq 1 \text { for } q_{3} \geq 2
\end{gathered}
$$

Thus, we see that $q=\operatorname{Max}\left(q_{1}, q_{2}, q_{3}\right)=2$. Further more, we see that

$$
\begin{aligned}
& \alpha(1)=1, \alpha(2)=3, \alpha(3)=2 \\
& \vartheta(1)=1, \vartheta(2)=2, \vartheta(3)=3
\end{aligned}
$$

Here, we see that

$$
\begin{aligned}
& G_{1}=\left\{z_{1}\right\}, G_{2}=\left\{z_{1}, z_{3}\right\}, G_{3}=\{Z\} \\
& K_{1}=\left\{z_{1}\right\}, K_{2}=\left\{z_{1}, z_{2}\right\}, K_{3}=\{Z\}
\end{aligned}
$$

On space $Z=\left\{z_{1}, z_{2}, z_{3}\right\}$, assume $\mu$ is a measure such that

From Table 1, we know that

$$
\begin{aligned}
& \mu\left(G_{0}\right)=0, \mu\left(G_{1}\right)=0.5, \mu\left(G_{2}\right)=0.8, \mu\left(G_{3}\right)=1 \\
& \mu\left(K_{0}\right)=0, \mu\left(K_{1}\right)=0.5, \mu\left(K_{2}\right)=0.7, \mu\left(K_{3}\right)=1
\end{aligned}
$$

Based on Equations (15) and (16), one has the following equation:

$$
\begin{aligned}
E^{+}(x) & =\operatorname{Choq}_{\mu}\left(E^{+}\left(z_{1}\right), \ldots, E^{+}\left(z_{n}\right)\right) \\
& =\sum_{j=1}^{3}\left(f\left(\mu\left(G_{j}\right)\right)-f\left(\mu\left(G_{j-1}\right)\right)\right) E^{+}\left(z_{\alpha(j)}\right) \\
& =\sum_{j=1}^{3}\left(\mu\left(G_{j}\right)-\mu\left(G_{j-1}\right)\right) E^{+}\left(z_{\alpha(j)}\right) \\
& =(0.5)(1)+(0.3)(0.8)+(0.2)(0.4) \\
& =0.82
\end{aligned}
$$




$$
\begin{aligned}
E^{-}(x) & =\left(1-\left(\sum_{j=1}^{3}\left(f\left(\mu\left(K_{j}\right)\right)-f\left(\mu\left(K_{j-1}\right)\right)\right)\left(1-\left(E^{-}\left(z_{\vartheta(j)}\right)\right)^{2}\right)^{1 / 2}\right)^{2}\right)^{1 / 2} \\
& =\left(1-\left(\sum_{j=1}^{3}\left(\mu\left(K_{j}\right)-\mu\left(K_{j-1}\right)\right)\left(1-\left(E^{-}\left(z_{\vartheta(j)}\right)\right)^{2}\right)^{1 / 2}\right)^{2}\right)^{1 / 2} \\
& =\left(1-\left((0.5)\left(1-0^{2}\right)^{1 / 2}+(0.2)\left(1-0.3^{2}\right)^{1 / 2}+(0.3)\left(1-0.5^{2}\right)^{1 / 2}\right)^{2}\right)^{1 / 2} \\
& =0.31
\end{aligned}
$$

Hence, $\left\langle E^{+}(z), E^{-}(z)\right\rangle=\langle 0.82,0.31\rangle$. We see that $(0.82)^{2}+(0.31)^{2}<1$. Finally, we get an orthopair fuzzy grade by the proposed model with $q=2$.

The proposed model requires an alternative in the decision-making process as an orthopair fuzzy set. Each of the orthopair fuzzy grades of orthopair fuzzy set as a criteria, which consists of the hesitancy degree, non-membership degree and membership degree. Then, the Equations (15) and (16) are used to aggregate all orthopair fuzzy grades of an orthopair fuzzy set and get a orthopair fuzzy grade. Finally the Equation (14) is used to get a degree of the orthopair fuzzy grade, which can represent the calculation of refined expected value of this alternative.

Table 1. The information about this measure.

\begin{tabular}{ccccccccc}
\hline $\mathrm{T}$ & $\phi$ & $\left\{z_{1}\right\}$ & $\left\{z_{2}\right\}$ & $\left\{z_{3}\right\}$ & $\left\{z_{1}, z_{2}\right\}$ & $\left\{z_{1}, z_{3}\right\}$ & $\left\{z_{2}, z_{3}\right\}$ & $\{Z\}$ \\
\hline$\mu(T)$ & 0 & 0.5 & 0.3 & 0.4 & 0.7 & 0.8 & 0.8 & 1 \\
\hline
\end{tabular}

\section{Study Case}

Consider the following: a library wants to purchase a coffee machine. The manager has to decide what kind should be chosen. Now, assume there are several possible choices $E_{i}(i=1,2,3,4,5)$ can be chosen by the library and three attributes $y_{1}$ (price), $y_{2}$ (function) and $y_{3}$ (appearance) are taken into account. According to the experts, $E_{4}$ is the best choice. Now, we use the refined expected value decision rules [40] to make the decision.

Assume fuzzy sets will represent the alternatives $E_{i}(i=1,2,3,4,5)$ as follows:

$$
E_{i}=\left\langle y_{j}, E_{i}\left(y_{j}\right)\right\rangle \text { and } j=1 \text { to } 3
$$

where, $E_{i}\left(y_{j}\right)$ represent the degree to which $E_{i}$ satisfies to $y_{j}$.

Then, according to the previous description, the five alternatives with fuzzy sets transformed into matrix as follows:

$$
E=\left[\begin{array}{c}
E_{1} \\
E_{2} \\
E_{3} \\
E_{4} \\
E_{5}
\end{array}\right]=\left[\begin{array}{lll}
\left\langle y_{1}, 0.4\right\rangle & \left\langle y_{2}, 0.8\right\rangle & \left\langle y_{3}, 0.5\right\rangle \\
\left\langle y_{1}, 0.5\right\rangle & \left\langle y_{2}, 0.3\right\rangle & \left\langle y_{3}, 0.6\right\rangle \\
\left\langle y_{1}, 0.9\right\rangle & \left\langle y_{2}, 0.8\right\rangle & \left\langle y_{3}, 0.3\right\rangle \\
\left\langle y_{1}, 0.7\right\rangle & \left\langle y_{2}, 0.1\right\rangle & \left\langle y_{3}, 0.4\right\rangle \\
\left\langle y_{1}, 0.9\right\rangle & \left\langle y_{2}, 0.4\right\rangle & \left\langle y_{3}, 0.6\right\rangle
\end{array}\right]
$$

Furthermore, we see that matrices consists of indexes of five alternatives. 


$$
\alpha=\left[\begin{array}{lll}
\alpha_{11} & \alpha_{12} & \alpha_{13} \\
\alpha_{21} & \alpha_{22} & \alpha_{23} \\
\alpha_{31} & \alpha_{32} & \alpha_{33} \\
\alpha_{41} & \alpha_{42} & \alpha_{43} \\
\alpha_{51} & \alpha_{52} & \alpha_{53}
\end{array}\right]=\left[\begin{array}{lll}
2 & 3 & 1 \\
3 & 1 & 2 \\
1 & 2 & 3 \\
1 & 3 & 2 \\
1 & 3 & 2
\end{array}\right]
$$

The probability of this matrix is as follows:

$$
\begin{aligned}
& \operatorname{Prob}\left(E_{1}=y_{1}\right)=0.5, \operatorname{Prob}\left(E_{1}=y_{2}\right)=0.4, \operatorname{Prob}\left(E_{1}=y_{3}\right)=0.1 \\
& \operatorname{Prob}\left(E_{2}=y_{1}\right)=0.2, \operatorname{Prob}\left(E_{2}=y_{2}\right)=0.4, \operatorname{Prob}\left(E_{2}=y_{3}\right)=0.4 \\
& \operatorname{Prob}\left(E_{3}=y_{1}\right)=0.2, \operatorname{Prob}\left(E_{3}=y_{2}\right)=0.3, \operatorname{Prob}\left(E_{3}=y_{3}\right)=0.5 \\
& \operatorname{Prob}\left(E_{4}=y_{1}\right)=0.6, \operatorname{Prob}\left(E_{4}=y_{2}\right)=0.2, \operatorname{Prob}\left(E_{4}=y_{3}\right)=0.2 \\
& \operatorname{Prob}\left(E_{5}=y_{1}\right)=0.3, \operatorname{Prob}\left(E_{5}=y_{2}\right)=0.4, \operatorname{Prob}\left(E_{5}=y_{3}\right)=0.3
\end{aligned}
$$

Then, the probabilities of subsets of $y_{i}$ as follows:

$$
\begin{aligned}
& \operatorname{Prob}_{1}\left(H_{1}\right)=0.4, \operatorname{Prob}_{1}\left(H_{2}\right)=0.5, \operatorname{Prob}_{1}\left(H_{3}\right)=1 \\
& \operatorname{Prob}_{2}\left(H_{1}\right)=0.4, \operatorname{Prob}_{2}\left(H_{2}\right)=0.6, \operatorname{Prob}_{2}\left(H_{3}\right)=1 \\
& \operatorname{Prob}_{3}\left(H_{1}\right)=0.2, \operatorname{Prob}_{3}\left(H_{2}\right)=0.5, \operatorname{Prob}_{3}\left(H_{3}\right)=1 \\
& \operatorname{Prob}_{4}\left(H_{1}\right)=0.6, \operatorname{Prob}_{4}\left(H_{2}\right)=0.8, \operatorname{Prob}_{4}\left(H_{3}\right)=1 \\
& \operatorname{Prob}_{5}\left(H_{1}\right)=0.3, \operatorname{Prob}_{5}\left(H_{2}\right)=0.6, \operatorname{Prob}_{5}\left(H_{3}\right)=1
\end{aligned}
$$

Hence, we get the refined expected values of these alternatives as follows:

$$
\begin{aligned}
E V_{r}\left(E_{1}\right) & =\sum_{i=1}^{3}\left(\left(\operatorname{Prob}_{1}\left(H_{i}\right)\right)^{r}-\left(\operatorname{Prob}_{1}\left(H_{i-1}\right)\right)^{r}\right) y_{i} \\
& =\left(\left(\operatorname{Prob}_{1}\left(H_{1}\right)\right)^{r}\right) y_{2}+\left(\left(\operatorname{Prob}_{1}\left(H_{2}\right)\right)^{r}-\left(\operatorname{Prob}_{1}\left(H_{1}\right)\right)^{r}\right) y_{3}+\left(\left(\operatorname{Prob}_{1}\left(H_{3}\right)\right)^{r}-\left(\operatorname{Prob}_{1}\left(H_{2}\right)\right)^{r}\right) y_{1} \\
& =(0.4) 0.8+((0.5)-(0.4)) 0.5+((1)-(0.5)) 0.4 \\
& =0.57
\end{aligned}
$$

The same is true:

$$
E V_{r}\left(E_{2}\right)=0.46, E V_{r}\left(E_{3}\right)=0.57, E V_{r}\left(E_{4}\right)=0.52, E V_{r}\left(E_{5}\right)=0.61
$$

From the discussion above, one get that $V\left(E_{5}\right)>V\left(E_{1}\right)=V\left(E_{3}\right)>V\left(E_{4}\right)>V\left(E_{2}\right)$. Then, we can find the choice $E_{5}$ is the best choice in this problem. It means that the refined expected value decision rules can not make a true decision in this issue.

Now, we use the refined expected value decision rules under orthopair fuzzy environment to make the decision. Assume orthopair fuzzy sets will represent the alternatives $E_{i}(i=1,2,3,4,5)$ as follows:

$$
E_{i}=\left\langle y_{j}, E_{i}^{+}\left(y_{j}\right), E_{i}^{-}\left(y_{j}\right)\right\rangle_{q} \text { and } j=1 \text { to } 3
$$

where, $E_{i}^{+}\left(y_{j}\right)$ and $E_{i}^{-}\left(y_{j}\right)$ represent the degree to which $E_{i}$ satisfies to $y_{j}$ and that to which $E_{i}(i=1,2,3,4,5)$ does not satisfy $y_{j}$.

Then, according to the previous description, the five alternatives with orthopair fuzzy sets transformed into a matrix as follows: 


$$
E=\left[\begin{array}{c}
E_{1} \\
E_{2} \\
E_{3} \\
E_{4} \\
E_{5}
\end{array}\right]=\left[\begin{array}{lll}
\left\langle y_{1}, 0.7,0.5\right\rangle & \left\langle y_{2}, 0.6,0.2\right\rangle & \left\langle y_{3}, 0.8,0.4\right\rangle \\
\left\langle y_{1}, 0.3,0.3\right\rangle & \left\langle y_{2}, 0.6,0.2\right\rangle & \left\langle y_{3}, 0.8,0.1\right\rangle \\
\left\langle y_{1}, 0.4,0.6\right\rangle & \left\langle y_{2}, 0.7,0.4\right\rangle & \left\langle y_{3}, 0.9,0.2\right\rangle \\
\left\langle y_{1}, 0.8,0.6\right\rangle & \left\langle y_{2}, 0.7,0.1\right\rangle & \left\langle y_{3}, 0.9,0.4\right\rangle \\
\left\langle y_{1}, 0.9,0.3\right\rangle & \left\langle y_{2}, 0.8,0.2\right\rangle & \left\langle y_{3}, 0.1,0.7\right\rangle
\end{array}\right]
$$

It is easy to get that $q=2$. Further more, we see that matrices consists of indexes of five alternatives.

$$
\begin{aligned}
& \alpha=\left[\begin{array}{lll}
\alpha_{11} & \alpha_{12} & \alpha_{13} \\
\alpha_{21} & \alpha_{22} & \alpha_{23} \\
\alpha_{31} & \alpha_{32} & \alpha_{33} \\
\alpha_{41} & \alpha_{42} & \alpha_{43} \\
\alpha_{51} & \alpha_{52} & \alpha_{53}
\end{array}\right]=\left[\begin{array}{lll}
3 & 1 & 2 \\
3 & 2 & 1 \\
3 & 2 & 1 \\
3 & 1 & 2 \\
1 & 2 & 3
\end{array}\right] \\
& \vartheta=\left[\begin{array}{lll}
\vartheta_{11} & \vartheta_{12} & \vartheta_{13} \\
\vartheta_{21} & \vartheta_{22} & \vartheta_{23} \\
\vartheta_{31} & \vartheta_{32} & \vartheta_{33} \\
\vartheta_{41} & \vartheta_{42} & \vartheta_{43} \\
\vartheta_{51} & \vartheta_{52} & \vartheta_{53}
\end{array}\right]=\left[\begin{array}{lll}
2 & 3 & 1 \\
3 & 2 & 1 \\
3 & 2 & 1 \\
2 & 3 & 1 \\
2 & 1 & 3
\end{array}\right]
\end{aligned}
$$

On $Y=\left\{y_{1}, y_{2}, y_{3}\right\}$, assume $\mu$ is a measure such that

$$
\begin{aligned}
& \mu(\theta)=0, \mu\left(y_{1}, y_{2}\right)=0.5 \\
& \mu\left(y_{1}\right)=0.2, \mu\left(y_{1}, y_{3}\right)=0.4 \\
& \mu\left(y_{2}\right)=0.4, \mu\left(y_{2}, y_{3}\right)=0.6 \\
& \mu\left(y_{3}\right)=0.3, \mu(Y)=1
\end{aligned}
$$

Here, relying on the matrix $\alpha$ and $\vartheta$ and $\mu$, we see that

$$
\begin{gathered}
G=\left[\begin{array}{lll}
G_{11} & G_{12} & G_{13} \\
G_{21} & G_{22} & G_{23} \\
G_{31} & G_{32} & G_{33} \\
G_{41} & G_{42} & G_{43} \\
G_{51} & G_{52} & G_{53}
\end{array}\right]=\left[\begin{array}{lll}
\left\{y_{2}\right\} & \left\{y_{2}, y_{3}\right\} & \{Y\} \\
\left\{y_{3}\right\} & \left\{y_{2}, y_{3}\right\} & \{Y\} \\
\left\{y_{3}\right\} & \left\{y_{2}, y_{3}\right\} & \{Y\} \\
\left\{y_{2}\right\} & \left\{y_{2}, y_{3}\right\} & \{Y\} \\
\left\{y_{1}\right\} & \left\{y_{1}, y_{2}\right\} & \{Y\}
\end{array}\right] \\
K=\left[\begin{array}{llll}
K_{11} & K_{12} & K_{13} \\
K_{21} & K_{22} & K_{23} \\
K_{31} & K_{32} & K_{33} \\
K_{41} & K_{42} & K_{43} \\
K_{51} & K_{52} & K_{53}
\end{array}\right]=\left[\begin{array}{lll}
\left\{y_{2}\right\} & \left\{y_{2}, y_{3}\right\} & \{Y\} \\
\left\{y_{3}\right\} & \left\{y_{2}, y_{3}\right\} & \{Y\} \\
\left\{y_{3}\right\} & \left\{y_{2}, y_{3}\right\} & \{Y\} \\
\left\{y_{2}\right\} & \left\{y_{2}, y_{3}\right\} & \{Y\} \\
\left\{y_{2}\right\} & \left\{y_{1}, y_{2}\right\} & \{Y\}
\end{array}\right]
\end{gathered}
$$

Relying on Equations (15) and (16), one has the following equation:

$$
\begin{aligned}
E=\{ & \left\langle E_{1}, 0.67,0.388\right\rangle,\left\langle E_{2}, 0.54,0.226\right\rangle,\left\langle E_{3}, 0.64,0.458\right\rangle, \\
& \left.\left\langle E_{4}, 0.77,0.433\right\rangle,\left\langle E_{5}, 0.47,0.537\right\rangle\right\}
\end{aligned}
$$

Relying on Equation (14) and assuming $\lambda$ is 0.5 , one has the following results.

From Table 2, one get that $V\left(E_{4}\right)>V\left(E_{2}\right)>V\left(E_{1}\right)>V\left(E_{3}\right)>V\left(E_{5}\right)$. Then, we can find that $E_{4}$ is the best choice in this problem. It means that the proposed model is validity in issue of decision making and that refined expected value decision rules under orthopair fuzzy environment is more efficient than the refined expected value decision rules. 
Table 2. The information about this measure.

\begin{tabular}{cccccc}
\hline $\mathbf{x}$ & $\boldsymbol{E}_{\mathbf{1}}$ & $\boldsymbol{E}_{\mathbf{2}}$ & $\boldsymbol{E}_{\mathbf{3}}$ & $\boldsymbol{E}_{4}$ & $\boldsymbol{E}_{5}$ \\
\hline$V(x)$ & 0.641 & 0.667 & 0.593 & 0.676 & 0.467 \\
\hline
\end{tabular}

\section{Conclusions}

This paper proposes the refined expected value decision rules under orthopair fuzzy environment. The proposed model requires an alternative in the decision-making process as an orthopair fuzzy set. Each of the orthopair fuzzy grades of this orthopair fuzzy set as a criteria, which consists of the membership degree, the non-membership degree and hesitancy degree. Then, some equations are used to aggregate all orthopair fuzzy grades of an orthopair fuzzy set and get a orthopair fuzzy grade. Finally the Equation (14) is used to get a degree of the orthopair fuzzy grade, which can represent the calculation of refined expected value of this alternative. The proposed model applies the refined expected value decision rules on decision making that is described by orthopair fuzzy environment, which means that the proposed model can enlarge the applied scope of the classical refined expected value decision rules. In conclusion, the proposed model can apply the refined expected value decision rules on orthopair fuzzy environment. Numerical examples verify the availability and flexibility of the proposed model.

Author Contributions: Methodology, Y.X. and Y.D.; writing—original draft, Y.X.; writing—review and editing, Y.D. All authors have read and agreed to the published version of the manuscript.

Funding: The work is partially supported by the National Natural Science Foundation of China (Grant No. 61973332).

Acknowledgments: The authors greatly appreciate the discussion of Yangxue Li.

Conflicts of Interest: The authors declare no conflict of interest.

\section{References}

1. Yuan, R.; Tang, M.; Wang, H.; Li, H. A Reliability Analysis Method of Accelerated Performance Degradation Based on Bayesian Strategy. IEEE Access 2019, 7, 169047-169054. [CrossRef]

2. Li, H.; Yuan, R.; Fu, J. A reliability modeling for multi-component systems considering random shocks and multistate degradation. IEEE Access 2019, 7, 168805-168814. [CrossRef]

3. Borzooei, R.; Sabetkish, M.; Roh, E.; Kologani, M.A. Int-Soft Filters in Hoops. Int. J. Fuzzy Log. Intell. Syst. 2019, 19, 213-222. [CrossRef]

4. Khademan, S.; Zahedi, M.M.; Jun, Y.B.; Borzooei, R.A. Fuzzy soft positive implicative hyper BCK-ideals in hyper BCK-algebras. J. Intell. Fuzzy Syst. 2019, 36, 2605-2613. [CrossRef]

5. Qian, H.M.; Huang, H.Z.; Li, Y.F. A novel single-loop procedure for time-variant reliability analysis based on Kriging model. Appl. Math. Model. 2019, 75, 735-748. [CrossRef]

6. Sun, C.; Li, S.; Deng, Y. Determining Weights in Multi-Criteria Decision Making Based on Negation of Probability Distribution under Uncertain Environment. Mathematics 2020, 8, 191. [CrossRef]

7. Borzooei, R.; Shenavaei, M.; Di Nola, A.; Zahiri, O. On EMV-Semirings. Math. Slovaca 2019, 69, 739-752. [CrossRef]

8. Mi, J.; Li, Y.; Beer, M.; Broggi, M.; Cheng, Y. Importance measure of probabilistic common cause failures under system hybrid uncertainty based on Bayesian network. Eksploat. I Niezawodn.-Maint. Reliab. 2020, 22, 112-120. [CrossRef]

9. Jun, Y.B.; Song, S.Z.; Kim, S.J. Distances between hyper structures and length fuzzy ideals of BCK/BCI-algebras based on hyper structures. J. Intell. Fuzzy Syst. 2018, 35, 2257-2268. [CrossRef]

10. Yager, R.R.; Reformat, M.Z.; To, N.D. Drawing on the iPad to input fuzzy sets with an application to linguistic data science. Inf. Sci. 2019, 479, 277-291. [CrossRef]

11. Xiao, F.; Ding, W. Divergence measure of Pythagorean fuzzy sets and its application in medical diagnosis. Appl. Soft Comput. 2019, 79, 254-267. [CrossRef] 
12. Gou, X.; Liao, H.; Xu, Z.; Herrera, F. Double hierarchy hesitant fuzzy linguistic term set and MULTIMOORA method: A case of study to evaluate the implementation status of haze controlling measures. Inf. Fusion 2017, 38, 22-34. [CrossRef]

13. Song, S.Z.; Bordbar, H.; Jun, Y.B. A new type of hesitant fuzzy subalgebras and ideals in BCK/BCI-algebras. J. Intell. Fuzzy Syst. 2017, 32, 2009-2016. [CrossRef]

14. Pan, Y.; Zhang, L.; Li, Z.; Ding, L. Improved Fuzzy Bayesian Network-Based Risk Analysis With Interval-Valued Fuzzy Sets and D-S Evidence Theory. IEEE Trans. Fuzzy Syst. 2019. [CrossRef]

15. Xiao, F. Generalization of Dempster-Shafer theory: A complex mass function. Appl. Intell. 2019. [CrossRef]

16. Pan, L.; Deng, Y. An association coefficient of belief function and its application in target recognition system. Int. J. Intell. Syst. 2020, 35, 85-104. [CrossRef]

17. Yager, R.R.; Petry, F.E. Using Quality Measures in the Intelligent Fusion of Probabilistic Information. In Information Quality in Information Fusion and Decision Making; Springer: Berlin/Heidelberg, Germany, 2019; pp. 51-77.

18. Liang, S.; Deng, X.; Jiang, W. Optimal data fusion based on information quality function. Appl. Intell. 2019, 49, 3938-3946. [CrossRef]

19. Liu, Q.; Tian, Y.; Kang, B. Derive knowledge of Z-number from the perspective of Dempster-Shafer evidence theory. Eng. Appl. Artif. Intell. 2019, 85, 754-764. [CrossRef]

20. Jiang, W.; Cao, Y.; Deng, X. A Novel Z-network Model Based on Bayesian Network and Z-number. IEEE Trans. Fuzzy Syst. 2019. [CrossRef]

21. liu, B.; Deng, Y. Risk Evaluation in Failure Mode and Effects Analysis Based on D Numbers Theory. Int. J. Comput. Commun. Control 2019, 14, 672-691.

22. Wen, T.; Deng, Y. The vulnerability of communities in complex networks: An entropy approach. Reliab. Eng. Syst. Saf. 2020, 196, 106782. [CrossRef]

23. Li, M.; Xu, H.; Deng, Y. Evidential Decision Tree Based on Belief Entropy. Entropy 2019, 21, 897. [CrossRef]

24. Yager, R.R. A class of belief structures based on possibility measures. Soft Comput. 2018, 22, 7909-7917. [CrossRef]

25. Wang, T.; Wei, X.; Huang, T.; Wang, J.; Valencia-Cabrera, L.; Fan, Z.; Pérez-Jiménez, M.J. Cascading Failures Analysis Considering Extreme Virus Propagation of Cyber-Physical Systems in Smart Grids. Complexity 2019, 2019. [CrossRef]

26. Liu, W.; Wang, T.; Zang, T.; Huang, Z.; Wang, J.; Huang, T.; Wei, X.; Li, C. A fault diagnosis method for power transmission networks based on spiking neural $\mathrm{P}$ systems with self-updating rules considering biological apoptosis mechanism. Complexity 2020, 2020, 2462647. [CrossRef]

27. Habib, S.; Garg, H.; Nie, Y.; Khan, F.M. An Innovative Approach towards Possibility Fuzzy Soft Ordered Semigroups for Ideals and Its Application. Mathematics 2019, 7, 1183. [CrossRef]

28. Yager, R.R. Generalized orthopair fuzzy sets. IEEE Trans. Fuzzy Syst. 2016, 25, 1222-1230. [CrossRef]

29. Wang, J.; Wang, P.; Wei, G.; Wei, C.; Wu, J. Some power Heronian mean operators in multiple attribute decision-making based on q-rung orthopair hesitant fuzzy environment. J. Exp. Theor. Artif. Intell. 2019, 1-29. [CrossRef]

30. Liu, P.; Liu, W. Multiple-attribute group decision-making method of linguistic q-rung orthopair fuzzy power Muirhead mean operators based on entropy weight. Int. J. Intell. Syst. 2019, 34, 1755-1794. [CrossRef]

31. Wang, P.; Wang, J.; Wei, G.; Wei, C. Similarity measures of q-rung orthopair fuzzy sets based on cosine function and their applications. Mathematics 2019, 7, 340. [CrossRef]

32. Liu, P.; Wang, P. Multiple-attribute decision-making based on Archimedean Bonferroni Operators of q-rung orthopair fuzzy numbers. IEEE Trans. Fuzzy Syst. 2018, 27, 834-848. [CrossRef]

33. Wei, G.; Gao, H.; Wei, Y. Some q-rung orthopair fuzzy Heronian mean operators in multiple attribute decision making. Int. J. Intell. Syst. 2018, 33, 1426-1458. [CrossRef]

34. Wang, J.; Wei, G.; Lu, J.; Alsaadi, F.E.; Hayat, T.; Wei, C.; Zhang, Y. Some q-rung orthopair fuzzy Hamy mean operators in multiple attribute decision-making and their application to enterprise resource planning systems selection. Int. J. Intell. Syst. 2019, 34, 2429-2458. [CrossRef]

35. Wei, G.; Wei, C.; Wang, J.; Gao, H.; Wei, Y. Some q-rung orthopair fuzzy maclaurin symmetric mean operators and their applications to potential evaluation of emerging technology commercialization. Int. J. Intell. Syst. 2019, 34, 50-81. [CrossRef] 
36. Wang, J.; Gao, H.; Wei, G.; Wei, Y. Methods for multiple-attribute group decision making with q-rung interval-valued orthopair fuzzy information and their applications to the selection of green suppliers. Symmetry 2019, 11, 56. [CrossRef]

37. Wang, P.; Wei, G.; Wang, J.; Lin, R.; Wei, Y. Dual hesitant q-Rung orthopair fuzzy hamacher aggregation operators and their applications in scheme selection of construction project. Symmetry 2019, 11, 771. [CrossRef]

38. Gou, X.; Liao, H.; Wang, X.; Xu, Z.; Herrera, F. Consensus based on multiplicative consistent double hierarchy linguistic preferences: Venture capital in real estate market. Int. J. Strateg. Prop. Manag. 2020, 24, 1-23. [CrossRef]

39. Zhou, Q.; Mo, H.; Deng, Y. A New Divergence Measure of Pythagorean Fuzzy Sets Based on Belief Function and Its Application in Medical Diagnosis. Mathematics 2020, 8, 142. [CrossRef]

40. Yager, R.R. Refined expected value decision rules. Inf. Fusion 2018, 42, 174-178. [CrossRef]

41. Wang, T.; Wang, J.; Ming, J.; Sun, Z.; Wei, C.; Lu, C.; Pérez-Jiménez, M.J. Application of neural-like P systems with state values for power coordination of photovoltaic/battery microgrids. IEEE Access 2018, 6, 46630-46642. [CrossRef]

42. Tan, Z.X.; Cheong, K.H. Cross-issue solidarity and truth convergence in opinion dynamics. J. Phys. A Math. Theor. 2018, 51, 355101. [CrossRef]

43. Wang, T.; Wei, X.; Huang, T.; Wang, J.; Peng, H.; Pérez-Jiménez, M.J.; Valencia-Cabrera, L. Modeling fault propagation paths in power systems: A new framework based on event SNP systems with neurotransmitter concentration. IEEE Access 2019, 7, 12798-12808. [CrossRef]

44. Gao, X.; Deng, Y. Quantum Model of Mass Function . Int. J. Intell. Syst. 2020, 35, 267-282. [CrossRef]

45. Dzitac, I.; Filip, F.G.; Manolescu, M.J. Fuzzy logic is not fuzzy: World-renowned computer scientist Lotfi A. Zadeh. Int. J. Comput. Commun. Control 2017, 12, 748-789. [CrossRef]

46. Liu, Y.; Jiang, W. A new distance measure of interval-valued intuitionistic fuzzy sets and its application in decision making. Soft Comput. 2019, 23. [CrossRef]

47. Gao, S.; Deng, Y. An evidential evaluation of nuclear safeguards. Int. J. Distrib. Sens. Netw. 2019, 15. [CrossRef]

48. Xiao, F. EFMCDM: Evidential fuzzy multicriteria decision making based on belief entropy. IEEE Trans. Fuzzy Syst. 2019. [CrossRef]

49. Wang, T.; Liu, W.; Zhao, J.; Guo, X.; Terzija, V. A rough set-based bio-inspired fault diagnosis method for electrical substations. Int. J. Electr. Power Energy Syst. 2020, 119, 105961. [CrossRef]

50. Song, Y.; Deng, Y. A new soft likelihood function based on power ordered weighted average operator. Int. J. Intell. Syst. 2019, 34, 2988-2999. [CrossRef]

51. Wang, C.; Tan, Z.X.; Ye, Y.; Wang, L.; Cheong, K.H.; Xie, N.G. A rumor spreading model based on information entropy. Sci. Rep. 2017, 7, 1-14. [CrossRef]

52. Xiao, F. A new divergence measure for belief functions in D-S evidence theory for multisensor data fusion. Inf. Sci. 2019. [CrossRef]

53. Gao, X.; Deng, Y. The Pseudo-Pascal Triangle of Maximum Deng Entropy. Int. J. Comput. Commun. Control 2020, 15, 1006. [CrossRef]

54. Deng, W.; Deng, Y. Entropic methodology for entanglement measures. Phys. A Stat. Mech. Its Appl. 2018, 512, 693-697. [CrossRef]

55. Tan, Z.X.; Koh, J.M.; Koonin, E.V.; Cheong, K.H. Predator Dormancy is a Stable Adaptive Strategy due to Parrondo's Paradox. Adv. Sci. 2019, 7, 1901559. [CrossRef] [PubMed]

56. Tan, Z.X.; Cheong, K.H. Nomadic-colonial life strategies enable paradoxical survival and growth despite habitat destruction. Elife 2017, 6, e21673. [CrossRef]

57. Wei, B.; Xiao, F.; Shi, Y. Fully Distributed Synchronization of Dynamic Networked Systems with Adaptive Nonlinear Couplings. IEEE Trans. Cybern. 2019, 1-9. [CrossRef]

58. Fei, L.; Zhang, Q.; Deng, Y. Identifying influential nodes in complex networks based on the inverse-square law. Phys. A Stat. Mech. Its Appl. 2018, 512, 1044-1059. [CrossRef]

59. Wang, H.; Fang, Y.P.; Zio, E. Risk Assessment of an Electrical Power System Considering the Influence of Traffic Congestion on a Hypothetical Scenario of Electrified Transportation System in New York Stat. IEEE Trans. Intell. Transp. Syst. 2019. [CrossRef]

60. Xu, P.; Zhang, R.; Deng, Y. A Novel Visibility Graph Transformation of Time Series into Weighted Networks. Chaos Solitons Fractals 2018, 117, 201-208. [CrossRef] 
61. Wei, B.; Xiao, F.; Shi, Y. Synchronization in Kuramoto Oscillator Networks with Sampled-Data Updating Law. IEEE Trans. Cybern. 2019, 1-9. [CrossRef]

62. Mo, H.; Deng, Y. Identifying node importance based on evidence theory in complex networks. Phys. A Stat. Mech. Its Appl. 2019. [CrossRef]

63. Yager, R.R.; Alajlan, N.; Bazi, Y. Uncertain database retrieval with measure-based belief function attribute values. Inf. Sci. 2019, 501, 761-770. [CrossRef]

64. Jin, L.; Mesiar, R.; Yager, R.R. Melting probability measure with OWA operator to generate fuzzy measure: The Crescent Method. IEEE Trans. Fuzzy Syst. 2018, 27, 1309-1316. [CrossRef]

65. Romagnoli, S. A vague multidimensional dependency structure: Conditional versus Unconditional fuzzy copula models. Inf. Sci. 2019, 512, 1202-1213. [CrossRef]

66. Garg, H. Novel neutrality operation-based Pythagorean fuzzy geometric aggregation operators for multiple attribute group decision analysis. Int. J. Intell. Syst. 2019, 34, 2459-2489. [CrossRef]

67. Shafer, G. A Mathematical Theory of Evidence; Princeton University Press: Princeton, NJ, USA, 1976; Volume 42.

68. Gou, X.; Xu, Z.; Liao, H.; Herrera, F. Multiple criteria decision making based on distance and similarity measures under double hierarchy hesitant fuzzy linguistic environment. Comput. Ind. Eng. 2018, 126, 516-530. [CrossRef]

69. Adler, A.M.; Calvo, T.; Mayor, G. Aggregation Operators; Sringer: Heidelberg, Germany, 2002.

70. Choquet, G. Theory of capacities. Annales de l'institut Fourier 1954, 5, 131-295. [CrossRef]

71. George, J.K.; Bo, Y. Fuzzy Sets and Fuzzy Logic: Theory and Applications; PHI: New Delhi, India, 1995; pp. $443-455$.

72. Hussain, A.; Ali, M.I.; Mahmood, T.; Munir, M. q-Rung orthopair fuzzy soft average aggregation operators and their application in multicriteria decision-making. Int. J. Intell. Syst. 2020, 35, 571-599. [CrossRef]

73. Li, H.; He, Y.; Nie, X. Structural reliability calculation method based on the dual neural network and direct integration method. Neural Comput. Appl. 2018, 29, 425-433. [CrossRef]

74. Garg, H.; Kumar, K. Linguistic interval-valued Atanassov intuitionistic fuzzy sets and their applications to group decision-making problems. IEEE Trans. Fuzzy Syst. 2019, 27, 2302-2311. [CrossRef]

75. Li, N.; Garg, H.; Wang, L. Some Novel Interactive Hybrid Weighted Aggregation Operators with Pythagorean Fuzzy Numbers and Their Applications to Decision Making. Mathematics 2019, 7, 1150. [CrossRef]

76. Fei, L.; Deng, Y. Multi-criteria decision making in Pythagorean fuzzy environment. Appl. Intell. 2020, 50, 537-561. [CrossRef]

77. Bai, K.; Zhu, X.; Zhang, R.; Gao, J. Linguistic Reasoning Petri Nets Using q-Rung Orthopair Fuzzy Linguistic Sets and Weighted Ordered Weighted Averaging Operators. IEEE Access 2019, 7, 103167-103183. [CrossRef]

78. Du, W.S. Research on arithmetic operations over generalized orthopair fuzzy sets. Int. J. Intell. Syst. 2019, 34, 709-732. [CrossRef]

79. Hussain, A.; Irfan Ali, M.; Mahmood, T. Covering based q-rung orthopair fuzzy rough set model hybrid with TOPSIS for multi-attribute decision making. J. Intell. Fuzzy Syst. 2019, 37, 981-993. [CrossRef]

80. Luqman, A.; Akram, M.; N Al-Kenani, A. q-Rung orthopair fuzzy hypergraphs with applications. Mathematics 2019, 7, 260. [CrossRef]

81. Luqman, A.; Akram, M.; Davvaz, B. q-Rung orthopair fuzzy directed hypergraphs: A new model with applications. J. Intell. Fuzzy Syst. 2019, 37, 3777-3794. [CrossRef]

82. Yager, R.R.; Alajlan, N.; Bazi, Y. Aspects of generalized orthopair fuzzy sets. Int. J. Intell. Syst. 2018, 33, $2154-2174$. [CrossRef]

83. Yager, R.R. Extending Set Measures to Pythagorean Fuzzy Sets. Int. J. Fuzzy Syst. 2019, $21,343-354$. [CrossRef]

(C) 2020 by the authors. Licensee MDPI, Basel, Switzerland. This article is an open access article distributed under the terms and conditions of the Creative Commons Attribution (CC BY) license (http:/ / creativecommons.org/licenses/by/4.0/). 